\title{
PBI: Entre el fetiche y ser uno de los mayores inventos del siglo $\mathrm{xx}$
}

\author{
GDP: between fetishism and one of the greatest inventions \\ of the 20th century
}

Santiago Dutto*

\footnotetext{
* Licenciado en Desarrollo, Facultad de Ciencias Sociales, Universidad de la República, Uruguay. $\bowtie$ duttosantiago@gmail.com https://orcid.org/0000-00022937-0801
}

RECIBIDO: [28.3.2018]

ACEPTADO: [9.5.2018]

\section{Resumen}

El PBI es un indicador económico. Varios elementos históricos y políticos lo convirtieron en una herramienta con la cual se evalúa el nivel de desarrollo de los países. Además se lo utiliza para considerar cuestiones relativas a la asignación de recursos y la ayuda internacional. El PBI falla como traductor del nivel de desarrollo, pero también tiene otras falencias de carácter técnico. Los defensores de su uso destacan, por un lado, que el indicador no se propone medir el desarrollo, por lo que no debe ser criticado en ese aspecto. Para algunos, el PBI puede mostrar una potencialidad de desarrollo por cuanto la dimensión económica se relaciona con otras dimensiones del desarrollo. Por otro lado, señalan que el indicador se ha corregido a lo largo de las décadas y ha incorporado varias de las críticas que se le han hecho.

Palabras clave: indicadores de desarrollo, desarrollo económico y social, análisis económico.

\section{Abstract}

The GDP is an economic indicator. Several historical and political issues turned it into a tool to assess countries' development levels. It is also used to consider aspects related to the allocation of resources and international 
cooperation. However, GDP fails as a "translator" of the levels of development, and, as we will see throughout this work, it also shows other technical flaws. Advocates of its use highlight, on the one hand, that the indicator does not intend to measure development, so it should not be criticized in that regard. For some, the GDP can show development "potential", since the economic dimension is related to other dimensions of development. On the other hand, they point out that the indicator has been rectified over the decades, responding to the criticisms that have been levelled at it.

Keywords: development indicators, economic and social development, economic analysis.

\section{Introducción}

El producto bruto interno (PBI) per cápita es uno de los indicadores más conocidos y utilizados del mundo. Si bien fue creado para medir actividad económica, su resultado se asoció rápidamente al bienestar o al nivel de desarrollo de un país.

En el año 2000 fue catalogado por la Oficina de Análisis Económico de Estados Unidos como «uno de los mayores inventos del siglo XX» (Lepenies, 2016a); sin embargo, existen quienes reclaman que se deje de utilizar, a la luz de las transformaciones en la economía. Otros abogan por utilizarlo con algunas correcciones y junto con un mayor número de indicadores, tanto económicos como de otras dimensiones del desarrollo.

La descripción que la Oficina de Análisis Económico de Estados Unidos hace del PBI afirma que su medición sirve para conocer, entre otros aspectos, cuán rápido crece la economía y cuáles son los patrones de gasto de los hogares (Constanza. Hart, Kubiszewski y Talberth, 2014).

El indicador efectivamente ha evolucionado y mejorado, teniendo en cuenta sus propias falencias técnicas, algunas de las críticas recibidas y un gran número de transformaciones del objeto que se propone medir. Pese a ello, muchas críticas subsisten y otras muchas se les han sumado, fruto de cambios en el paradigma de desarrollo y las nuevas concepciones del bienestar.

Aunque desde su creación se indicó que el resultado del PBI no debía considerarse una medida del bienestar, distintos sucesos históricos lo han ubicado en ese lugar.

Veremos entonces algunos aspectos históricos, así como las principales críticas y deficiencias. 


\section{Breve historia del PBI}

Coyle (2014) refiere que en 1665, en Gran Bretaña, un científico creó un concepto rudimentario de cuentas nacionales con el propósito de cuantificar los ingresos y gastos de Inglaterra y Gales, así como la cantidad de tierra, población y algunos recursos, para conocer si eran capaces de solventar un conflicto armado que se avecinaba.

Este tipo de empresa fue también llevado adelante por otros países, pero siempre de manera precaria y con enormes diferencias tanto en los elementos incluidos como en forma en que se incluían. Algunos economistas clásicos aportaron luego, sobre esta base, elementos relativos a la producción material e inmaterial. A finales del siglo XIX un trabajo seminal de Alfred Marshall incluyó los servicios, con lo que, según algunos autores, inauguró el sistema de cuentas nacionales moderno (Coyle, 2014).

El indicador - como se lo conoce ahora, aunque con algunas variaciones- fue creado en los Estados Unidos entre las décadas del treinta y el cuarenta. En esos años el mundo vivía aún años de turbulencia económica y social caracterizados por dos guerras mundiales y la Gran Depresión (Constanza et al., 2014). A su vez, a diferencia de lo que había sucedido hasta entonces, medir la economía se convertía en algo importante porque nunca había crecido al ritmo con que lo hacía en ese momento.

Los autores explican que el presidente Theodore Roosevelt utilizaba el PBI para tranquilizar al pueblo estadounidense. Explicaba que su nivel de vida no se vería amenazado frente a la inminente participación del país en la Segunda Guerra Mundial, ya que la producción sería suficiente para la nueva empresa.

En 1944, 44 naciones del bando de los aliados se reunieron en Bretton Woods para crear un proceso de cooperación internacional que permitiera regular el sistema monetario y financiero, a fin de sanear algunos de los problemas que se habían generado al final de la guerra.

Entre los productos de la conferencia se destaca la creación del Fondo Monetario Internacional y el Banco Internacional de Reconstrucción y Fomento, ahora parte del Banco Mundial.

Dichos organismos internacionales debían dar voz y colocar en su agenda a todos los países que los integran. Sin embargo, debido al equilibrio de fuerzas resultante de la guerra, Estados Unidos dominó ambas instituciones.

Esto llevó a que los estándares estadounidenses —como su moneda, su economía o sus políticas económicas - se convirtieran en la vara con la cual se medía al resto de los países (Constanza et al., 2014). Más adelante se verá que ello tiene su proceso análogo en el paradigma de desarrollo, con una visión del desarrollo econocentrista y eurocentrsita, en la cual las instituciones de los países desarrollados son el faro que debe guiar el desarrollo del resto del mundo y sus políticas se convierten en recetas mágicas. 
Como corolario, y en lo que más nos atañe, dada la participación de Estados Unidos y Gran Bretaña en la creación del PBI, su rol en la Bretton Woods devino en que los nuevos organismos utilizarían el indicador como principal herramienta para evaluar las economías y el desarrollo de todos los países socios.

La reestructuración de estas instituciones sobre los años setenta hizo perder participación a Estados Unidos, pero no cambió su rol ni su estatus sobre el PBI. Los setenta trajeron un gran número de crisis. Coyle (2014) menciona el estancamiento del crecimiento económico en un gran número de países, combinado con altos niveles de inflación. La intensidad de la Guerra Fría, las voces que reclamaban por la sustentabilidad de la producción y los fracasos en la eficiencia de la ayuda internacional completan el cuadro.

La importancia de estos eventos radica en que desataron críticas al paradigma que regía la economía y sus instituciones, y que había gozado de tanto prestigio desde el fin de la Segunda Guerra. Una de esas instituciones era la concepción que se tenía sobre la forma de medir el éxito económico y en especial sobre el rol del PBI.

En los años noventa, un nuevo embate contra la capacidad del PBI llegó con la revolución informática y se centró en la (in)capacidad de medir la innovación. A esto se sumó el conjunto de voces que dudaban de qué tan bien el PBI lograba contabilizar los servicios.

Coyle indica que, frente a algunas de estas críticas, países como Estados Unidos modificaron la metodología. Estas modificaciones no superan los límites de lo que el indicador se propone medir, pero intentan dar cuenta de cambios en la economía. Por ejemplo, el software es ahora contabilizado como una inversión (Coyle, 2014, p. 89), y eso conlleva importantes variaciones en el resultado de la medición. También ha habido mejoras en la medición de servicios.

En los últimos años ha sido el sistema financiero el que ha planteado mayor cantidad de dudas respecto a la capacidad del PBI de incluirlo correctamente en sus mediciones.

\section{El indicador}

El PBI per cápita de un país es un estimado de todo aquello que produce el país en un período dado, casi siempre un año, dividido por la cantidad de habitantes de dicho país. Se calcula sumando el consumo de bienes y servicios de los hogares, los gastos del Estado, y el saldo de la balanza comercial y de la cuenta capital.

Por su facilidad de construcción, comparación y comunicación, el PBI se ha convertido en uno de los principales indicadores de la actividad económica. Una de las razones por las cuales es tan criticado como indicador del desarrollo no es realmente 
culpa del indicador, sino de quienes conciben el desarrollo como crecimiento o como desarrollo económico. Constanza, Hart, Posner y Talberth (2009) indican que el PBI no es inherentemente malo, sino que «mide lo que mide».

Cuando hablamos de que el PBI presenta limitaciones nos referimos a dos aspectos separados: por un lado, las limitaciones técnicas del indicador referidas a la construcción del dato; por otro, las limitaciones para relevar o presentar el bienestar de un país.

Fleurbaey (2009) explica que existe una larga tradición de buscar la relación entre el bienestar social y el consumo o el ingreso total, y el PBI per cápita ha sido el indicador por antonomasia de la perspectiva económica del bienestar. Sus defensores se amparan en la objetividad del indicador como su principal cualidad, pese a los constantes embates que ha sufrido por quienes sostienen que no logra medir efectivamente lo que se propone. Philipp Lepenies (2016a) va un poco más lejos e indica que el PBI es la figura estadística más poderosa de la historia.

Respecto a este rol, Antal y Van der Bergh (2014) sostienen que es el hábito del uso lo que ha asociado el PBI a la idea de bienestar y desarrollo, pero que eso no tiene respaldo científico en ninguna teoría macroeconómica PBI.

Stiglitz (2009), por su parte, habla de «fetichismo» por el uso del indicador y advierte que, en tiempos en que se pondera el desempeño, la forma en que se miden las cosas orienta las decisiones sobre qué hacer.

En cuanto al poder que ha tenido el indicador, Van den Bergh (2008) advierte que los gobiernos invierten enormes sumas de dinero para calcular y predecir el PBI. Este elemento es común a todos los países y ha llegado a ser tomado y estandarizado en el Sistema de Cuentas Nacionales de las Naciones Unidas, lo que permite hacer comparaciones basadas en el PBI. Si el PBI no tuviese validez o significancia, implicaría una enorme pérdida de dinero. Asimismo, afirma que el indicador es utilizado por tomadores de decisión como un input de vital importancia.

Además, el conformismo, la docilidad, la socialización y la imitación pueden explicar por qué el PIB es sin mucha crítica aceptado por la mayoría de los ciudadanos y estudiantes de economía por igual. Esto es ayudado por el repetido énfasis de la relevancia del PIB en la educación y en los medios de comunicación públicos, incluyendo periódicos, televisión, radio e internet. (Van den Bergh, 2008, p. 120)

Si bien el rol ahora corresponde al PBI per cápita, a mitad del siglo XX ese lugar era ocupado por el stock de capital. Los autores más reconocidos de la época que escribían sobre el crecimiento y el desarrollo encontraban en la inversión en infraestructura la condición sine qua non para alcanzar el crecimiento que luego generaría de- 
rrames hacia el resto de la sociedad, lo que permitiría alcanzar el desarrollo. Autores como Rostow (1960), Lewis (1954), Nurkse (1961) y Hirshmann (1958), inscriptos en dicha literatura, concebían una receta para el desarrollo según la cual, una vez subsanada la falta de capital - única limitación del sistema一, se iniciaba un círculo virtuoso que conducía a la concreción del desarrollo.

El desarrollo puede comprenderse en un sentido general como un proceso de cambio orientado a aumentar el bienestar de las personas (Bertoni et al., 2011). Si bien hoy el concepto trasciende ampliamente la dimensión económica del proceso, que a su vez se ha alejado del mero crecimiento económico, el crecimiento del PBI sigue siendo la meta que supuestamente esconde todas las soluciones mágicas para los problemas del desarrollo.

Seers (1969) indica que el PBI per cápita puede, a lo sumo, ser una medida de potencialidad del desarrollo. Se refiere con esto a que muchas de las metas intermedias o umbrales en otras dimensiones del desarrollo pueden ser conseguidas por medios económicos. Y Phillip Lepenies (2016b) afirma que el PBI es una medida fundamentalmente materialista, donde el único imperativo es producir una mayor cantidad de bienes y servicios sin tener en cuenta si ello afecta el bienestar de las personas.

Según José Antonio Alonso (2013), para algunos países las limitantes del desarrollo son ahora trampas del desarrollo o mecanismos por los cuales, pese a que cuentan con los medios económicos, sus instituciones o infraestructuras no les permiten alcanzar el desarrollo. Para otros países la situación es incluso peor, ya que existen dichas trampas y a su vez no cuentan con los cuantiosos recursos económicos que supone transformar aspectos clave, como la matriz productiva, o hacer grandes inversiones en infraestructura.

Si bien el paradigma del desarrollo se ha transformado a lo largo de estas últimas décadas para cambiar su visión economicista por una visión holística, muchas instituciones aún lo conciben como una relación cuasilineal entre el tamaño de la economía y la capacidad de avanzar en el resto de las dimensiones, con lo que validan el PBI per cápita como indicador de desarrollo.

Es el caso, por ejemplo, del papel que desempeña en la asignación de la ayuda oficial al desarrollo (AOD). La AOD es un mecanismo de la OCDE para otorgar préstamos en condiciones favorables a países en desarrollo. Para indicar qué países son pasibles de integrar la lista de receptores de AOD, la OCDE utiliza los umbrales de renta generados por el Banco Mundial, cuya construcción se basa únicamente en el PBI per cápita.

Son varios los autores que se han proclamado contrarios al uso del indicador como medida asociada a cualquier tipo de actividad de evaluación del desarrollo, ya sea como indicador de desempeño o como herramienta para la asignación o creación de políticas. 
Joseph Stiglitz, premio Nobel de Economía en 2001, ha cuestionado duramente el indicador en numerosos trabajos. En su trabajo del 2009 en la Comisión Sobre la Medición del Desarrollo Económico y el Progreso Social (Stiglitz, Sen y Fitoussi, 2009) se afirma que la correlación entre el PBI y otros indicadores económicos tiende a hacerse más débil a medida que se estudia por sectores específicos, y que el indicador puede llevar a inferencias incorrectas sobre el bienestar de los individuos.

Coyle (2014) discrepa con Stiglitz et al. (2009) respecto a la aseveración de que «el PBI principalmente mide producción de mercado»; intercambia los factores e indica que el PBI define mercado y luego lo mide. Este autor es partidario de ahondar en la discusión sobre de qué hablamos cuando hablamos de economía en el siglo XXI.

\section{Las críticas}

A continuación detallaremos algunas de las principales limitaciones del PBI per cápita. El eje de la revisión será siempre el PBI como indicador del desarrollo o de la dimensión económica del desarrollo.

Es importante dividir las críticas en dos grupos. Por una parte están aquellas referidas al modo en que se construye el PBI, para las cuales las limitaciones técnicas pueden ser subsanadas sin cuestionar la idoneidad técnica del indicador para medir el desarrollo. Por otra parte están las críticas que se centran en las limitaciones del paradigma sobre el cual se construye el indicador, para las cuales las propuestas superadoras se basan en no utilizar el indicador o en hacerle cambios radicales para que se acerque a medir la dimensión económica del desarrollo en su totalidad.

Si bien a priori puede ser injusto criticar al indicador por sus falencias en algo que no se propone hacer, el rol que ha asumido ha llegado a tal grado de validación que justifica dejar bien en claro todo lo que el PBI no mide, aun cuando pueda asumirse como un indicador del desarrollo.

En los últimos años han sido varios los autores (Antal y Van den Bergh, 2014; Van den Bergh, 2009; Stiglitz et al., 2009) cuyos trabajos recopilan críticas al PBI per cápita.

Comenzamos a describir entonces las principales críticas respecto a la metodología del indicador de PBI per cápita.

\section{Medida bruta}

El PBI es primeramente una medida bruta. Esto quiere decir que no toma la depreciación del capital al indicar su valor. Cuando queremos hablar de bienestar, y sobre todo 
al asociarlo al desarrollo, es importante destacar el papel que desempeñan el stock de capital y los ciclos de inversión para los países.

El producto en estado bruto es leído como una posibilidad de transformación perfecta entre producto y bienestar. Cuando el enfoque es el de desarrollo, sin embargo, si gran parte del producto debe ser utilizada para reinvertir en maquinaria, esta trasformación dista de ser perfecta.

Una solución para esta limitación es el empleo de una medida neta. Como advertíamos al comienzo, eso complejiza la construcción del indicador. Si bien el producto interno neto guarda mayor coherencia con lo que se busca estimar, la empresa que supone medirlo suele ser de tal magnitud que lleva a desistir del intento.

\section{Consumo e ingreso en vez de producto}

La tradición que busca una relación entre bienestar e ingreso o consumo tiene al menos unas cuantas décadas y se basa en un consenso mayor que el que genera el uso del PBI per cápita, según indica Fleurbaey (2009). El autor explica que dicha solución se ve aquejada por algunos problemas, como el de definir el signo y la magnitud en los cambios en el nivel de bienestar, así como definir correctamente el nivel de bienestar.

Si bien consumo e ingreso evalúan el bienestar de forma indirecta y son menos fieles que los indicadores sobre autopercepción de bienestar, permiten medir de mejor manera la capacidad de los individuos y los hogares de hacer efectivas sus posibilidades de consumo. Es importante destacar que en la gran mayoría de los índices seguimos trabajando con datos del promedio del consumo, por lo que es importante evaluarlos siempre a la luz de los ratios de pobreza y los niveles de desigualdad.

Algunos indicadores basados en el PBI pero con correcciones ya tienen en cuenta esta falencia y ajustan el resultado teniendo en cuenta flujos de rentas de extranjeros en el país, o de nacionales en el exterior, o la depreciación del capital.

Si bien los resultados entre el PBI y algunos de los indicadores corregidos no presentan grandes diferencias para los países desarrollados, el tipo de matriz productiva y las complejas relaciones históricas y socioeconómicas de los países menos desarrollados los hacen más proclives a presentar mayores discrepancias.

\section{Precios de mercado}

El PBI únicamente refleja los precios de mercado de los bienes transados en dichos mercados. Antal y Van den Bergh (2014) advierten, a su vez, que la medida estima los costos y no los beneficios de los productos. 
Por otra parte, el paradigma actual de desarrollo concibe que sean fundamentales muchos elementos que no se transen en el mercado, incluso cuando sean bienes tangibles. Para estos últimos, se les da un precio de mercado a determinados bienes utilizando distintos proxis, según el bien y la información con la que se cuente.

Stiglitz et al. (2009) afirman que para Francia en 2007 casi un tercio del consumo final de los hogares respondía a imputaciones de precios, ya que estaba inscripto en categorías tales como consumo de bienes provistos por el Estado o autoproducción.

Los autores indican que «las imputaciones vienen con un precio». Por un lado, tenemos la exactitud del dato y el hecho de que para imputar un valor se deben crear varios supuestos, lo que hace al dato menos confiable. Por otro, las distintas imputaciones no son igualmente fáciles de comprender $\mathrm{y}$, aunque la cuenta de autoproducción sea sencillamente considerada como ingreso, «por ejemplo, las personas pueden no reconocer el valor de los servicios de vivienda ocupados por el propietario como ingresos» (Stiglitz et al., 2009, p. 90).

También Antal y Van der Bergh (2014) señalan la incapacidad del PBI para medir el bienestar real de todos los productos transados en el mercado, ya que su concepción lo acerca a una medida de costos de dichos productos. Incluso advierten que la medición del PBI no se basa en una buena gestión de libros contables.

A su vez, y como corolario de dicha crítica, los autores sostienen que el PBI per cápita no logra captar todos los costos sociales y omite las externalidades. Estos dos elementos son de suma importancia cuando se aduce que el PBI es una buena medida del bienestar o del nivel de desarrollo de un país. Las externalidades y los costos sociales tampoco se distribuyen equitativamente. A su vez, es importante criticar el uso del PBI per cápita si presenta una imagen de desarrollo o bienestar sin salvaguardas pero omite los elementos negativos de cómo se genera ese producto.

\section{Consideraciones intertemporales}

Algunos autores critican bajo este título la relación entre el PBI y el bienestar, porque si esta relación guardara alguna especie de linealidad, un crecimiento sostenido acumulado del PBI por muchos de años también debería figurar en los reportes de bienestar.

Antal y van den Bergh hacen una simple cuenta e indican que un crecimiento sostenido del $2 \%$ anual por un lapso de 1000 años significaría aproximadamente un incremento de cuatrocientos millones de veces el PBI actual, y que es casi imposible visualizar que el bienestar pueda comportarse de igual manera. 


\section{Preferencias lexicográficas y bienestar subjetivo}

Las preferencias lexicográficas hacen referencia a una teoría económica que intenta explicar la manera en que un individuo se enfrenta a las decisiones y posibilidades de consumo. La teoría indica que existe una jerarquía entre las necesidades primarias, convencionales y personales, y que dichas necesidades dan base al sistema que ordena las preferencias de un individuo. A su vez, no todas las necesidades son tangibles y no se puede satisfacer una necesidad de un grupo consumiendo un bien de otro grupo (Dancourt, 1986).

Si esto se aplica al PBI a la hora de medir el bienestar, la crítica es que el indicador no logra dar cuenta de que no es posible una sustitución perfecta del bienestar que proveen los bienes transables por el que proveen los bienes no transables. Van den Bergh (2008) utiliza esta terminología para indicar por qué el PBI per cápita solo puede dar cuenta del valor de mercado de los bienes materiales que suponen a su consumidor algún grado de utilidad y bienestar, pero no puede tomar en cuenta todo aquello que, si bien genera bienestar poseerlo, no puede ser comprado. El autor ejemplifica esta idea con elementos como la serenidad, el aire limpio o el acceso a la naturaleza.

Marc Fleurbaey (2009) indica que subsanar esa limitación generaría una suerte de PBI corregido que, junto con la depredación del medio ambiente, se haría eco de dos aspectos centrales en las críticas sin poder corregir todas las demás.

Aunque muchas de las críticas recopiladas en este apartado guardan alguna conexión con una visión de desarrollo, en el siguiente se hace mayor hincapié en los aspectos referidos al desarrollo y a la dimensión económica del bienestar que el PBI no logra medir correctamente o que omite por completo.

\section{Disponibilidad del producto}

Al relevar las críticas al PBI per cápita como indicador del desarrollo se destaca que una de las principales carencias es la diferencia entre producción y disponibilidad de determinado producto. (Si bien esta crítica podría ser ubicada dentro del grupo de elementos que el PBI no mide, la incluimos a continuación de «Medida bruta» porque refiere también a una porción de la producción de la cual no se dispone.)

Imaginemos que hemos logrado medir de manera aceptable la depreciación del capital. Nos enfrentamos ahora a la realidad de que no todo lo que se produce en un país queda en mano de las empresas o los individuos de ese país o en las arcas de ese Estado. El producto nacional disponible (PND) se propone entonces dar cuenta de cuánto de lo producido por el país o en el mundo es pasible de ser utilizado por dicho país. 
Para los países en la periferia de las relaciones económicas, el PBI o el producto nacional neto (PNN) pueden dar una idea de producción mucho más grande que aquello de lo que efectivamente disponen y que podría luego, mediante los mecanismos correctos, trasladarse a procesos de desarrollo o de mejora del bienestar.

A los países desarrollados esta realidad no les es ajena, pero con algunas salvedades: por un lado, es posible que un país desarrollado con una industria que remite ganancias a otro país desarrollado cuente con empresas o industrias que hagan lo mismo para su beneficio. Por otro, las protecciones en materia de aranceles, control de inversiones e impuestos colocan a estos países en mejores posiciones para hacerse de porcentajes más altos de esos beneficios, o al menos para generar procesos de adopción de capacidades y conocimientos que los países en desarrollo no siempre pueden crear por tener menor capacidad de presión.

\section{Economía informal}

El fenómeno de la economía informal, indica Van der Bergh (2008), existe tanto en los países en desarrollo como en los desarrollados y se relaciona con actividades tan diferentes como la agricultura de subsistencia, el trabajo voluntario, las tareas domésticas y de cuidado de niños. Una de las mayores falencias del PBI es que no considera las actividades informales y el bienestar que estas pueden generar. El autor indica que el crecimiento del PBI puede a veces responder a la formalización de un sector preexistente en la economía, por lo que se sobrestima el bienestar si se tienen en cuenta cambios en el PBI.

En una línea similar sobre actividades que no son reflejadas en el producto de un país, la Unión Europea viene debatiendo desde hace varios años sobre si las actividades ilegales, como la prostitución (donde sea ilegal) o el tráfico de drogas, deben ser incluidas en sus cuentas, ya que para algunos países podrían llegar a representar aumentos en su producto del 1 al 5\%. En España, por ejemplo, en el 2014 las actividades ilegales aportaron al PBI 9200 millones de euros al ser incluidas en las cuentas nacionales por el INE.

\section{Externalidades y recursos naturales}

Existe una suerte de lógica perversa en la forma en que se computa el PBI y su relación con el medio ambiente.

En cuanto a los sistemas productivos, el indicador no castiga el desempeño si un país decide llevar adelante la extracción o explotación de sus recursos naturales sin 
tener en cuenta la capacidad de carga del ecosistema o la sostenibilidad de la producción. Los países pueden entonces ser alentados a aumentar el producto sin considerar el bienestar presente o el futuro.

Stiglitz et al. (2009) dan el ejemplo de que un país con muchos atascos en el tránsito podría aumentar su PBI si la demanda de gasolina crece por ser utilizada de manera ineficiente; sin embargo, esto claramente no aumenta el bienestar. En la misma línea podemos imaginar un país que ve cómo crece su PBI al llevar adelante un proceso de reconstrucción después de un desastre natural.

Por otro lado, el producto de un país no indica por sí solo de qué manera se produce; es decir, omite los aspectos negativos de la producción a todo nivel. Tomando en cuenta todos los elementos que considera la definición de desarrollo, esta crítica es una de las más fuertes a la utilización del indicador.

Así como los aspectos concernientes a la distribución, la igualdad y las oportunidades fueron conformándose un espacio fundamental en la concepción del desarrollo, la protección del medio ambiente es el eje en torno al cual se concibe el desarrollo como generador de prácticas sustentables que permitan pensar en las generaciones futuras.

El PBI que se utiliza actualmente no toma en cuenta la degradación del medio ambiente cuando se llevan a cabo actividades económicas. Sobre algunas consideraciones generales al momento de plantear mejoras a la medición del aspecto económico del bienestar, Ribarsky, Kang y Bolton (2016) proponen hacer mayor hincapié en la perspectiva de los hogares y no de los individuos. Este punto también es uno de los ejes de la Comisión para la Medición del Desempeño Económico y el Bienestar Social (Stiglitz, et al., 2009) y de muchos autores críticos del PBI como indicador del bienestar.

\section{Tecnología y matriz productiva}

Respecto a la tecnología y la matriz productiva, tenemos dos posibles interpretaciones en cuanto a sus aspectos negativos. Por un lado existe una degradación del medio debido a la utilización de tecnología que no es de punta o por el tipo de modelo de explotación económica. Por otro, los aspectos referidos al tipo de relaciones sociales, económicas y políticas que se dan en los países caracterizados por economías primarizadas o extractivistas y sus roles en la economía mundial.

Si bien la tecnología y la matriz productiva son conceptos asociados, no son sinónimos. Cuando hablamos de tecnología podemos evocar la problemática en la que ahondábamos al principio del artículo, sobre la necesidad de utilizar medidas netas para visualizar de mejor manera los costos que conlleva la depreciación del capital, entendida como aquella porción del producto que no se logra convertir en bienestar. 
En cuanto a la matriz productiva, nos referimos a la cantidad, la calidad y el tamaño de los distintos sectores de la economía. Este último aspecto ha sido revestido de una importancia fundamental al pensar el desarrollo de los países, ya que en estas últimas décadas se ha cuestionado duramente el discurso de que cualquier matriz productiva con las instituciones necesarias puede generar crecimientos sostenidos con un efecto derrame hacia la sociedad que permita un proceso sostenible de desarrollo.

Autores como Dani Rodrik (2005, 2007) o Ricardo Hausmann (2007) han estudiado la importancia de la composición de las canastas exportadas y la matriz productiva. El discurso no es nuevo: Raúl Presbich fue un gran impulsor de la tesis del subdesarrollo como proceso con causas externas a los países y la importancia de las dinámicas que se construyen en torno al centro y la periferia de la economía, dinámicas que a su vez generan mecanismos de autopreservación del sistema.

Si tomáramos únicamente el dato del PBI podríamos creer que países con niveles similares tienen iguales perspectivas hacia el futuro o igual desempeño en indicadores asociados al desarrollo.

Si bien ya trabajamos en torno a elementos como la depreciación y las externalidades, cada matriz productiva es un factor fundamental en el entretejido social y político de los países, por lo que las externalidades en el plano social también difieren entre países.

\section{«Estudios empíricos de bienestar» y bienestar subjetivo}

El primero de los elementos responde a una categoría de críticas mencionada por Antal y Van den Bergh, quienes explican brevemente que durante las décadas de 1950 a 1970 algunos estudios sobre reportes de felicidad permitían concluir que los niveles de bienestar se estancaban, o llegaban a disminuir, pese a que el PBI per cápita aumentaba.

Los autores encuentran la razón de dicho fenómeno en la teoría que indica que, al llegar a un nivel de ingreso dado, el costo del crecimiento excede el bienestar que genera.

En este apartado nombraremos brevemente aspectos referidos al bienestar subjetivo, por cuanto responde a un paradigma de desarrollo en el que se explicita que la dimensión económica es tan solo una de las múltiples variables que componen el bienestar, y que dentro de dicha dimensión existen, a su vez, aspectos más amplios que no conciernen al consumo.

Una de las principales críticas que se le hacen a la medición del bienestar, o del desarrollo, mediante indicadores indirectos es que en última instancia el nivel general de bienestar se intuye a partir de elementos como los ingresos económicos. En esta 
línea de crítica al PBI per cápita como indicador existe un enfoque sobre la subjetividad del bienestar. Si bien la validez la otorga el hecho de indicar cómo el individuo percibe y comunica su situación personal, los valores del indicador se correlacionan con el desempeño en otros indicadores objetivos de bienestar.

«Quienes hacen las políticas deberían tomar el bienestar subjetivo de la población como su meta y pensar en todos los demás bienes como la educación, la salud y el ingreso como medios para ese fin», afirma Richard Layard (2012, p. 22). El autor explica que, al dejar de utilizar el PBI como indicador de bienestar, la calidad de vida desde un punto de vista subjetivo es la forma más acertada de medir el bienestar.

McGillivray y Clarke (2006) señalan que el bienestar subjetivo involucra una evaluación multidimensional de la vida, lo que incluye juicios de la satisfacción con la vida y evaluaciones de las emociones y los estados de ánimo. Por su parte, Bruni y Porta (2007) indican que el bienestar subjetivo está constituido por cuatro componentes: a) emociones placenteras, b) emociones no placenteras, c) juicio global o evaluación sobre la vida y d) dominio de la satisfacción (matrimonio, salud, etcétera).

Estos autores indican, además, que la felicidad es un concepto más estrecho que el bienestar subjetivo y diferente de la satisfacción con la vida: aunque la felicidad y la satisfacción con la vida son componentes del bienestar subjetivo, la satisfacción con la vida refleja la percepción individual de la distancia con las aspiraciones, mientras que la felicidad es un balance entre efectos positivos y negativos sobre la vida. Bruni y Porta (2007) expresan que en este marco el bienestar subjetivo es sinónimo de felicidad, en concordancia con la concepción aristotélica de felicidad, en la que satisfacción y felicidad son consideradas según el criterio hedonista de sentirse feliz.

\section{Inflación y desarrollo}

La inflación es otro de los elementos económicos que no aparecen representados en el desempeño del PBI. Junto con el desempleo, la inflación es una de las variables más importantes al evaluar los reportes de autopercepción de bienestar.

Si bien son varios los elementos que no se reflejan en el producto de un país, la inflación se destaca por su impacto social y por la manera en que se refleja en el desarrollo.

La inflación es una suba generalizada de los precios al consumo. Su importancia radica, por un lado, en cómo afecta al bienestar de las personas (Shiller, 1997; Frey y Stutzer, 2001; Galli y Van der Hoeven, 2001) y, por otro, en cómo evoluciona en los países según su nivel de desarrollo. Sin embargo, son varios los autores que destacan una relación no lineal entre inflación y desarrollo, en la que se añaden elementos como la apertura económica o la calidad institucional de los bancos centrales. 
Cómo indica Octavio Rodríguez (2001), Raúl Prebisch planteaba ya en 1961 que la inflación de gastos e inversiones no es sino producto de la debilidad estructural de las economías en la fase de contracción y la incapacidad de los bancos centrales de contrarrestar el proceso en los ciclos de bonanza. Estas dinámicas terminan trasladando luego las presiones hacia los procesos inflacionarios latentes en los costos.

La inflación es un problema del desarrollo, dado que atenta de manera regresiva contra los salarios; es decir, aquellos individuos que perciben los salarios más bajos a menudo reciben ingresos de sectores que presentan mayores dificultades para acompasar sus remuneraciones a la suba de precios, y por tanto pierden capacidad real de consumo, lo que obviamente atenta contra su bienestar.

Aparicio y Araujo (2011) sostienen que niveles bajos y controlados de inflación no tienen carácter regresivo, porque este no solo depende de niveles altos de inflación que se dan en forma de shock, sino también de las desigualdades en la sociedad.

El indicador aislado, por fuera de la dinámica inflacionaria, solo puede brindar información muy limitada sobre el desempeño de la economía en su conjunto o el bienestar de un país. Sin embargo, por lo que puede significar para los individuos de menores ingresos y porque las dinámicas responden a cuestiones del desempeño de la matriz productiva, es importante tomarlo en cuenta en el conjunto de indicadores que reflejan la economía de un país.

\section{Desigualdad y pobreza}

El PBI no se propone medir, entre otras cosas, los niveles de pobreza o de desigualdad en un país. Estos dos elementos son de suma importancia al considerar la dimensión económica del desarrollo, no solo por su dimensión ética, sino porque la pobreza y la desigualdad generan a los países enormes costos económicos en forma de ineficiencia productiva, altos costos sociales y medioambientales por nombrar algunos.

En este sentido, el PBI no está construido para ponderar los resultados productivos del país en función de la desigualdad o los niveles de pobreza. La principal propuesta en torno a este punto es utilizar indicadores que se centren en la distribución del ingreso, el consumo o la riqueza.

Este cambio permitiría tener una mejor visión sobre el bienestar de cada individuo en función de qué lugar ocupa en las respectivas distribuciones; sin embargo, la fotografía de la dimensión económica del bienestar distaría de estar completa.

Antal y Van den Bergh (2014) opinan que el PBI per cápita representa el ingreso promedio, sin tener en cuenta la distribución, y por ello es inconsistente con la economía ortodoxa, para la cual la utilidad marginal del ingreso decrece a medida que este aumenta. 


\section{Inversión y ahorro}

La inversión y el ahorro son importantes indicadores para evaluar la salud y las perspectivas de las economías. Estos dos aspectos se refieren, en términos generales, a la autonomía de las economías para decidir los patrones de inversión y cómo posicionarse frente a decisiones de cambio en las matrices productivas.

Incluso los patrones de la inversión extranjera directa o los indicadores que toman en cuenta en qué invierten los países han cobrado mayor relevancia en los discursos sobre desarrollo, indicadores y sostenibilidad.

Tal es el caso del indicador ahorro genuino (AG). La importancia de este indicador radica en que uno de los principales elementos para su construcción es el concepto de desarrollo sostenible, entendido como aquel proceso de desarrollo en el cual el bienestar presente no se consigue amenazando el bienestar futuro o en el que el bienestar no disminuye a lo largo del tiempo (Hamilton, 2000).

Si bien la discusión en torno al concepto de sostenibilidad no es pertinente a este trabajo, en los últimos años se ha centrado en torno a la existencia de una sostenibilidad blanda y una fuerte. La primera, y la que usa el AG, entiende que la sostenibilidad de cada factor es intercambiable por la de otro - por ejemplo, recursos naturales y capital humano-, mientras que la sostenibilidad fuerte arguye que existen niveles mínimos y un stock de capital natural que no puede ser sustituido por otros factores en la ecuación.

El AG es el ahorro bruto de un país, en porcentaje de su PBI, corregido según la depreciación de capital, la inversión en educación, la relación con el medio ambiente con base en la extracción y el uso de recursos naturales y la contaminación (Antal y Van den Bergh, 2014).

\section{Principales medidas superadoras}

Si bien son varios los factores que explican el rol y el status del PBI, la facilidad de construcción e implementación es una de las fortalezas más destacadas. Por ello las medidas superadoras más aceptadas no esperan corregir el PBI sino crear nuevos indicadores que den cuenta de una visión holística del desarrollo, o al menos de elementos como la sostenibilidad del desarrollo, en sus varios niveles. 


\section{PBI verde}

A comienzos de este siglo, Asheim (2000) se cuestiona si un producto nacional neto sería capaz de servir como medida de bienestar o de indicar si el modelo de desarrollo actual es sustentable.

Las razones por las cuales es importante contar con un PBI verde son compartidas por quienes se abocan a su estudio, quienes destacan la importancia de tomar en cuenta no solo las cantidades producidas sino la forma en que se producen.

Asheim (2000, p. 1) indica que la contabilidad verde puede cumplir tres propósitos: «medir el ingreso de bienestar equivalente, el ingreso sustentable o el beneficio social neto» aunque los elementos responden siempre a la relación entre consumo y producción y la sustentabilidad del modelo.

Por otro lado, Talbert y Bohara (2006) plantean que al hacer hincapié en el PBI verde se retoma el debate sobre la relación entre apertura comercial y crecimiento. Los autores, que se encuentran entre los promotores de la apertura, sostienen que la contracara del proceso de globalización es que en el mundo ha aumentado la inequidad, debido sobre todo a la competencia para atraer inversiones, que ha generado desregulación en algunos sectores.

El PBI verde podría entonces, en palabras de los autores, «tender puentes» entre ambas discusiones, ya que dicho proxy recoge algunas críticas hechas al PBI (Talberth y Bohara, 2006, p. 745).

Dentro de los sistemas de PBI verde se destacan, entre otros, dos índices: el índice de bienestar económico sostenible (IBES) y el índice de progreso genuino o índice de progreso real (IPR).

El IPR revisa algunos aspectos que se le critican al PBI y toma en cuenta tanto la distribución de la renta como la deuda externa, sobre la base de que estos elementos son importantes para comprender de mejor manera el desempeño económico de un país en cuanto a su nivel de bienestar.

\section{El enfoque de las capacidades}

Amartya Sen fue uno de los primeros en explicar el desarrollo de las sociedades y el bienestar desde una perspectiva de capacidades de los individuos. Este foco en las personas significó un cambio de paradigma en cuanto a cómo se concebía el desarrollo. El enfoque de las capacidades provee un marco teórico del cual se han desprendido distintos tipos de índices, indicadores o corrientes teóricas para medir las capacidades de los individuos, que incluyen, excluyen o ponderan determinados aspectos por sobre otros. 
Análogamente a lo que sucede con las mediciones multidimensionales de bienestar, que cuestionan el rol que tiene el PBI al medir el bienestar, el enfoque de Sen cuestiona el rol del ingreso incluso como una correcta medida de pobreza.

El enfoque de las capacidades aduce que, a grandes rasgos, el bienestar se genera a partir de que un individuo es capaz de insertarse y llevar una vida dentro de la sociedad con base en un gran número de recursos diversos. Esto ha generado tanto defensores como detractores sobre el hecho de que el enfoque, a diferencia de sus antecesores, no conciba una relación lineal entre ingreso, riqueza y bienestar.

No existe un único indicador que cuente con la anuencia de todos quienes se pronuncian a favor del enfoque, sino varios índices o propuestas que destacan más o menos elementos en lo relativo a la privación de determinados derechos u oportunidades.

Sen (1985) formaliza algunos aspectos de su enfoque mediante una relación entre utilidad y libertad de hacer algo, lo que genera un set $\mathrm{Q}$ de capacidades $^{1}$ que da cuenta de una función donde el conjunto $Q$ de funciones factibles depende de las características de una persona y de sus derechos o acceso a los productos básicos.

Anand et al. (2009) indican que esto representa el set de capacidades de un individuo. De este enfoque parten algunas formalizaciones (Burchardt y Vizard, 2011; Anand et al., 2009; Anand y van Hees, 2006; Robeyns, 2011) que intentan medir las capacidades de los individuos y cómo efectivamente gozan o no de sus libertades. En este sentido, el enfoque de las capacidades suele presentar una marcada diferencia respecto a otras mediciones del bienestar, incluso multidimensional, debido a que aquel valora la libertad como algo efectivo pero también potencial.

Los aspectos incluidos suelen repetirse en los distintos trabajos y son varios los que distinguen entre un núcleo básico de capacidades y uno ampliado o que reviste mayor importancia una vez desarrolladas las capacidades básicas.

Burchardt y Vizard (2011), por ejemplo, toman una lista de diez capacidades fruto de un complejo proceso deliberativo llevado adelante por The Equality and $\mathrm{Hu}-$ man Rights Commission (EHRC) - para fiscalizar y monitorear la equidad y los derechos humanos en Gran Bretaña, concretamente: vida; salud; seguridad física; seguridad legal; educación y aprendizaje; estándar de vida; actividades productivas; participación; voz e influencia; vida individual, familiar y social; identidad; expresión y autorrespeto (p. 99). A su vez, son muchas las variables que componen cada uno de estos diez dominios, o los indicadores que pueden ser utilizados para definir si efectivamente un individuo goza de dichas capacidades. 
Anand et al. (2009) parten del núcleo de capacidades propuesto por Martha Nussbaum (2000), y mediante un trabajo de control estadístico generan 17 indicadores conectados a estas capacidades que explican en última instancia cambios en el bienestar de los individuos.

\section{Brechas}

El enfoque de brechas se basa principalmente en la concepción de que las realidades de los países en desarrollo se caracterizan por presentar problemáticas sumamente heterogéneas, no solo frente a países desarrollados sino que son diversas y distintas dentro del conjunto de los países en desarrollo.

La CEPAL desarrolla un enfoque alternativo «que implica incorporar explícitamente, en la agenda de cooperación para el desarrollo, la evaluación de necesidades y carencias que no están representadas por indicadores de ingresos sino que reflejan otro tipo de brechas» (CEPAL, 2012, p. 9).

El uso del concepto de brecha como aquello que falta o que el propio país no logra conseguir por sus propios medios no es nuevo: los primeros economistas que concebían al desarrollo como un progresivo alcance de metas consideraban que en las naciones subdesarrolladas existía una brecha de ahorro doméstico.

El análisis de las brechas financieras no es nuevo en el estudio del desarrollo internacional y tiene su origen en las propuestas económicas de los años 1950; el cálculo del 0,7 \% para la ayuda (que data de finales de los años sesenta) estaba basado en las brechas de ahorro y de divisas. (Alonso, Glennie y Sumner, 2014, p. 7)

El foco se coloca, entonces, no únicamente en la medición final, que revela si un país es desarrollado, sino en las dinámicas en las cuales presenta carencias o problemas que le impiden alcanzar mayores estadios de desarrollo. Es importante destacar que en dicho enfoque las brechas se presentan como elementos agudos y persistentes, que por diversas razones (económicas, políticas, culturales) no pueden ser subsanados únicamente por esfuerzos propios del país afectado, sino que para ello se requieren nuevas instituciones, capacidades técnicas, conocimientos o fondos.

José Antonio Alonso propone diferenciar entre brechas y trampas de desarrollo. Las primeras refieren a una incapacidad nacional de hacer frente a dichos problemas, mientras que las segundas conciernen a cómo se conjugan y relacionan determinados factores que en las presentaciones/interacciones actuales resultan en frenos para el desarrollo, aunque su superación no necesariamente requiere una gran cantidad de 
financiamiento externo. Estas trampas de desarrollo no son tan acuciantes como las trampas de pobreza que reflejan carencias absolutas, pero reflejan estrangulamientos que pueden convertirse en igual de limitantes en los procesos de desarrollo (Alonso, 2013).

\section{Índices multidimensionales}

Pese a que algunos índices, como el IDH, han perfeccionado su metodología a través de sucesivas construcciones, uno de los aspectos fundamentales, y a menudo el más criticado, es cómo se construye la agregación de las distintas variables que componen el total del índice. Veenhoven (2007) critica algunos de estos índices por tratarse de, como dice él, una agregación entre peras y manzanas.

El índice de desarrollo humano (IDH) es uno de los más utilizados como medida para el desarrollo desde una perspectiva no económica.

[...] es un índice compuesto que se centra en tres dimensiones básicas del desarrollo humano. La esperanza de vida al nacer refleja la capacidad de llevar una vida larga y saludable. Los años promedio de escolaridad y los años esperados de escolaridad reflejan la capacidad de adquirir conocimientos. Y el ingreso nacional bruto per cápita refleja la capacidad de lograr un nivel de vida digno. (PNUD, 2016, p. 3)

El índice de progreso social, construido por la organización Social Progress Imperative, «provee una medida basada en resultados, holística, objetiva y transparente del bienestar de un país que es independiente de indicadores económicos» (Stern, Wares y Hellman, 2016, p. 2). Los autores definen el progreso social como:

[...] la capacidad de la sociedad de satisfacer las necesidades humanas básicas de sus ciudadanos, estableciendo los bloques de construcción que permitan a los ciudadanos y las comunidades mejorar y sostener la calidad de sus vidas y crear las condiciones para que todas las personas alcancen todo su potencial. (Stern, Wares y Hellman, 2016, p. 2)

De dicha definición se desprenden entonces tres dimensiones: necesidades básicas, fundamentos del bienestar y oportunidades, cada una de las cuales tiene cuatro componentes. Necesidades básicas se divide en nutrición y cuidado médico básico; agua y sanidad; refugio/alojamiento y seguridad personal. Fundamentos del bienestar amplía la calidad de vida de los individuos y se compone de elementos que sirven de 
nexo con la dimensión siguiente: acceso al conocimiento básico; acceso a la información y las comunicaciones; salud y calidad medioambiental. Oportunidades refiere a la ampliación de derechos, capacidades y oportunidades según la concepción de desarrollo humano y comprende derechos personales; libertad personal y capacidad de elegir; tolerancia e inclusión, y acceso a educación avanzada.

\section{Conclusiones}

Si bien varias de las carencias o limitaciones técnicas del PBI han sido corregidas a lo largo de casi cien años de uso, e incluso se han generado nuevos indicadores que lo mejoran, varias de sus características criticadas aún persisten.

Las principales críticas técnicas responden a características de la medición, como el hecho de ser una medida bruta, el no poder dar cuenta de la disponibilidad del producto o la forma en la cual se imputan los precios, y cómo contempla la economía informal o incluso la ilegal.

Las críticas centradas en la medida de desarrollo destacan en el PBI la total ausencia de indicadores de desigualdad y de pobreza, así como la no consideración de la forma en que el producto se genera, en lo que se destacan dos elementos: las externalidades de la producción y la matriz productiva.

El PBI no fue concebido por sus creadores como un indicador de bienestar; sin embargo, diversas razones históricas, políticas y coyunturales le otorgaron un rol y un estatus que ha ostentado hasta el día de hoy.

El problema del PBI per cápita no radica únicamente en sus limitaciones técnicas, sino en el uso que se le da. Aunque el indicador como sinónimo de desarrollo tiene cada vez menos adeptos, a medida que las críticas se consolidan, sigue integrando mecanismos para evaluar cuestiones relativas al desarrollo realmente importantes. Por nombrar una: el nivel de PBI per cápita es la única base para crear los umbrales de renta con los cuales se condiciona la obtención de ayuda oficial al desarrollo.

La facilidad de construcción y comunicación se destacan como las principales fortalezas del PBI per cápita. Sin embargo, varios autores sostienen que esas ventajas no compensan sus debilidades ni los perjuicios que genera. Otros hablan de fetichismo por el uso del indicador y señalan que no existe consenso sobre hacia dónde avanzar cuando se habla de construir indicadores más fieles a los procesos de desarrollo.

Si bien es indiscutible que la construcción de políticas y la evaluación del estado de situación de los países requieren indicadores, el precio que se paga por la sencillez de construcción y aplicación es demasiado alto, por cuanto lleva a malinterpretar el nivel de desarrollo de los países y las limitaciones en cada dimensión. 
Mientras algunos autores abogan por erradicar el indicador cuando se habla de desarrollo, otros afirman que el producto del país, si es corregido en algunos aspectos técnicos, es al menos un buen marco de potencialidad para alcanzar el desarrollo.

Si bien el uso del PBI como medida de bienestar no tiene casi defensores, no existe el mismo nivel de acuerdo en cuanto a si su uso debería erradicarse ni sobre qué indicador debería ocupar su lugar.

En ese momento surgen con fuerza las voces que afirman que el PBI ha sido transformado a lo largo de los años y tiene un buen desempeño en la tarea que se propone llevar adelante: medir el crecimiento de la economía.

Si bien no existe consenso al respecto, autores como Coyle (2014) van más allá e invitan a reflexionar en torno a si lo que concebimos como aspectos centrales de la economía se han mantenido incambiados y si es eso lo que el PBI se propone medir.

Las Metas del Desarrollo Sostenible propuestas por la ONU avanzan hacia el paradigma que concibe las políticas para el desarrollo según las fortalezas y debilidades de cada país en cada una de las dimensiones del desarrollo.

La dimensión económica no puede ser medida con un único indicador, ya que contiene aspectos diversos y además no todos los países se comportan igual, aun cuando tengan niveles similares de producto.

Si bien la economía ha sufrido enormes cambios en el último siglo, y han surgido modelos y corrientes como la economía humana, que también han transformado el paradigma del desarrollo, el PBI ha mantenido su rol y estatus, con transformaciones bastante menores si las comparamos con el cambio que se le exige.

\section{Bibliografía}

Alonso, J. A. (2013). Cooperación con países de renta media: Un enfoque basado en incetivos. Madrid: AECID. (Documentos de Trabajo, 1).

Alonso, J. A., Glennie, J. y Sumner, A. (2014). Receptores y contribuyentes: Los países de renta media y el futuro de la cooperación para el desarrollo. Nueva York: DESA. (DESA Working Paper, 135).

Anand, P., Hunter, G., Carter, I., Dowding, K., Guala, F. y van Hees, M. (2009). «The development of capability indicators». Journal of Human Development and Capabilities, $10(1), 125-152$.

ANAND, P. y VAN HEES, M. (2006). «Capabilities and achievements: An empirical study». The Journal of Socio-Economics, 35(2), 268-284.

ANTAL, M. y VAN DEN BERGH, J. (2014). Evaluating alternatives to GDP as measures of social welfare/progress. Vienna: WWWforEurope. (Working Paper, 56). 
ApARICIO, C. y ARAujo, R. (2011). El impacto de la inflación en la distribución del ingreso: la importancia del nivel de desigualdad del ingreso inicial. Lima: Banco Central de Reserva del Perú. (Documentos de Trabajo, 004).

AsheIM, G. (2000). «Green national accounting: Why and how?». Environment and Development Economics, 5, 25-48.

Bertoni, R.; Castelnovo, C.; Cuello, A.; Fleitas, S.; Pera, S.; Rodríguez, J. y Rumeau, D. (2011). Construcción y análisis de problemas del desarrollo: ¿Qué es el desarrollo? ¿Cómo se produce? ¿Qué se puede hacer para promoverlo? Montevideo: UR-UCUR: CSE.

Bruni, L. y PoRTA, P. L. (2007). Handbook on the economics of happiness. Cheltenham: Edward Elgar.

BuRCHARDT, T. y VizARD, P. (2011). “"Operationalizing” the capability approach as a basis for equality and human rights monitoring in twenty first century Britain». Journal of Human Development and Capabilities, 12(1), 91-119.

CEPAL (2012). Los países de renta media: Un nuevo enfoque basado en brechas estructurales. Santiago de Chile: CEPAL.

CONSTANZA, R., HART, M., Kubiszewski, I. y TAlberth, J. (2014). «A short history of GDP: Moving towards better measures of human well-being». Solutions, 5, 91-97.

Constanza, R., Hart, M., Posner, S. y TAlBerth, J. (2009). Beyond GDP: The need for new measures of progress. Boston: Boston University. (The Pardee Papers, 4).

CoYLE, D. (2014). GDP: A brief but affectionate history. Princeton: Princeton University Press.

DANCOURT, O. (1986). «Restricción externa, economía de mercado y economía de guerra». Economía, 9(17-18), 133-148.

FleurbaeY, M. (2009). «Beyond GDP: The quest for a measure of social walfare». Journal of Economic Literture, 47(4), 1029 - 1075.

Frey, B. y StutZer, A. (2001). Happiness and economics. Princeton: Princeton University Press.

GALLI, R. y VAN DER HoEven, R. (2001). Is inflation bad for income inequality: The importance of the initial rate of inflation. Genève: ILO. (Employment paper, 29).

HAMILTON, K. (2000). Genuine savings as a sustainabilty Indicator. Washington: The World Bank.

HAUSMANN, R., HWANG, J. y RODRIK, D. (2007). «What you export matters». Journal of Economic Growth, 12(1), 1-25. doi: 10.1007/s10887-006-9009-4.

HiRSCHMAN, A. (1958). The strategy of economic development. New Haven: Yale University Press.

LAYARD, R. (2012). Why measure subjective well-being? Recuperado de http:// oecdobserver.org/news/fullstory.php/aid/3767/Why_measure_subjective_wellbeing_html. 
LEPENIES, P. (2016a). The power of a single number: A political history of GDP. Nueva York: Columbia University Press.

LEPENIES, P. (2016b). Why GDP? Recuperado de https://www.project-syndicate. org/commentary/why-gdp-by-philipp-lepenies-2016-08?a_la=english\&a_d= 57b31ac92bd5d215f04cf482\&a_m=\&a_a=click\&a_s=\&a_p=homepage\&a_li=whygdp-by-philipp-lepenies-2016-08\&a_pa=login-info-bookmarks\&a_ps=.

LEWIS, A. (1954). «Economic development with unlimited supplies of labor». The Manchester School, 22(2), 139-191.

McGillivray, M. y Clarke, M. (2006). «Human well-being: Concepts and measures». En M. McGillivray y M. Clarke, Understanding human well-being (pp. 3-15). Tokio: UNU Press.

NURKSE, R. (1961). Problems of capital formation in underdeveloped countries. Nueva York: Oxford University Press.

Nussbaum, M. (2000). Women and human development. Cambridge: Cambridge University Press.

PNUD (2016). Progreso multidimensional: Bienestar más allá del ingreso. Nueva York: PNUD.

RIBARSKY, J., KANG, C. y Bolton, E. (2016). The drivers of differences between growth in GDP and household adjusted disposable income in OECD countries. París: OECD Publishing. (OECD Statistics Working Papers, 06).

RoBEYNS, I. (2011). «Sen's capability approach and gender inequality: Selecting relevant capabilities». Feminist Economics, 9(2-3), 61-92.

Rodríguez, O. (2001). «Presbich: Actualidad de sus ideas básicas». Revista de la CEPAL, (75), 41-52.

RODRIK, D. (2005). «Políticas de diversificación económica». Revista de la CEPAL (87), 7 23.

Rostow, W. W. (1960). The stages of economic growth: A non-communist manifiesto. Londres: Cambridge University Press.

SEERS, D. (1969). The meaning of development. Brighton: Institute of Development Studies. (IDS Communication, 44).

SEN, A. (1985). Commodities and capabilities. Amsterdam: North-Holland.

SHILlER, R. (1997). «Why do people sislike inflation?». En C. Romer y D. Romer (eds.), Reducing inflation: Motivation and strategy (pp. 13-70). Chicago: University of Chicago Press.

Stern, S., Wares, A. y Hellman, T. (2016). Social Progress Index 2016: Methodological Report. Recuperado de http://13i8vn49fibl3go3i12f59gh.wpengine.netdnacdn.com/wp-content/uploads/2016/07/SPI-2016-Methodological-Report.pdf.

STiglitz, J. (2009). Fetichismo del PBI. Recuperado de https://www.projectsyndicate.org/commentary/gdp-fetishism/spanish. 
StiglitZ, J., SEn, A. y Fitoussi, J. P. (2009). Report by the Commisssion on the Measurement of Economic Performance and Social Progress. Recuperado de http://ec. europa.eu/eurostat/documents/118025/118123/Fitoussi+Commission+report. TAlBerth, J. y BOHARA, A. (2006). «Economic openness and green GDP». Ecological Economics, 58(4), 743-758.

VAN DEN BERGH, J. (2008). «The GDP paradox». Journal of Economic Psychology, 30(2), 117-135.

VeEnhoven, R. (2007). «Subjective measures of well-being». En M. McGillivray (ed.), Human well-being: Concept and measurement (pp. 214-239). Houndmills: Palgrave/McMillan. 